\title{
A Protection Algorithm Based on Transient Current Spectrum Characteristic for MMC-HVDC Line
}

\author{
Xingguo Wang \\ China Electric Power Research Institute, Haidian District, Beijing, China
}

\begin{abstract}
When a fault occurs on a transmission line, fault generated high frequency transient components are a series of natural frequencies in frequency domain. This paper proposes a protection algorithm for MMC-HVDC transmission line based on spectrum characteristic of natural frequency. Spectrum correlation coefficient is used to determine whether a fault is internal or external fault. The scheme performance was proven using PSCAD/EMTDC simulations in $\pm 500 \mathrm{kV}$ MMC-HVDC power system. The simulation results show that it can identify fault quickly and accurately.
\end{abstract}

Keywords-MMC-HVDC transmission line; natural frequency; protection; transient current; spectrum characteristic

\section{INTRODUCTION}

Power system protection has traditionally relied on the measurement of power frequency components for identifying internal and external faults, fault-generated high-frequency transient signals are considered as interference and filtered out [1]. However, these high-frequency components contain extensive fault information. In fact, these high-frequency transient signals contain more information about the fault than power frequency signals.

For the stability of the electric network, it is important to clear faults from transmission lines quickly with the aid of a high speed protection. The protection scheme based on fault transient signals will achieve fast detection time. The faultgenerated travelling wave information has been used to identify internal and external faults in transmission lines [2]-[8].

However, the scheme based on travelling wave may fail to detect faults under certain conditions [9]. Firstly, for traveling wave protection, the transducer used to measure voltage and current signals must have a large bandwidth since fault generated waveforms may contain high frequencies. Secondly, when phase-to-ground fault occurs near zero voltage level, the amplitude of wave fronts will be small and the relay might not be able to detect the fault. Thirdly, the traveling wave distance protection is to measure the time interval between the arrival of an incident wave towards the fault points and that of the corresponding wave reflected from it, but in some cases the second wave front is not from fault point and the protection might fail. Fourth, for a close-up fault, the time difference between the arrival of an incident wave and the arrival of its reflection from the busbar will be so short that the traveling wave fronts are unlikely to be detected separately.

In this paper, a protection algorithm based on spectrum characteristic of natural frequency component for MMC-
HVDC line was proposed. In Section II, the characteristics of natural frequency component are analyzed in frequency component. It is a series of natural frequencies in frequency domain. In Section III, a protection algorithm using spectrum correlation coefficient is proposed to determine whether a fault is internal or external to the protected zone. In Section IV, the protection scheme performance is tested extensively using PSCAD/EMTDC-generated data, the simulation verified that the proposed scheme can identify internal and external fault accurately. The results also show that the scheme is insensitive to fault position, fault inception angle, fault resistance.

\section{SPECTRUM CHARACTERISTIC OF NATURAL FREQUENCY COMPONENT}

\section{A. Fundamental Spectrum Characteristic of Natural Frequency Component}

The equivalent circuit of fault network in the Laplace domain using distributed parameter model is shown in Figure I, where $Z_{c}$ is surge impedance, $Z_{1}$ and $Z_{2}$ is source impedance and fault resistance, $E_{1}$ and $E_{2}$ is source and imposing source in fault position, $\mathrm{W}_{1}$ and $\mathrm{W}_{2}$ is equivalent source of traveling wave [10].

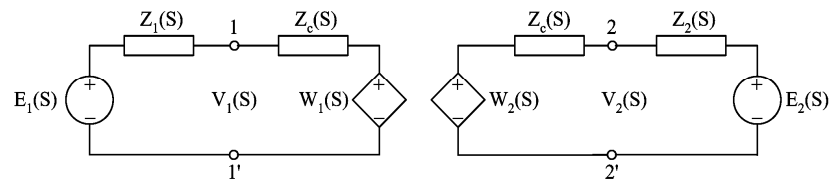

FIGURE I. EQUIVALENT CIRCUIT OF FAULT NETWORK USING DISTRIBUTED PARAMETER MODEL

The voltages in 1-1' and 2-2'can be expressed that

$$
\begin{aligned}
& V_{1}(s)=\frac{Z_{1}(s)}{Z_{1}(s)+Z_{C}(s)} W_{1}(s)+\frac{Z_{c}(s)}{Z_{1}(s)+Z_{C}(s)} E_{1}(s) \\
& V_{2}(s)=\frac{Z_{2}(s)}{Z_{2}(s)+Z_{C}(s)} W_{2}(s)+\frac{Z_{c}(s)}{Z_{2}(s)+Z_{C}(s)} E_{2}(s)
\end{aligned}
$$

where

$$
\begin{aligned}
W_{1}(s) & =\frac{P(s)}{1-\Gamma_{1}(s) \Gamma_{2}(s) P^{2}(s)} \\
\cdot & {\left[\Gamma_{2}(s) P(s) \frac{2 Z_{C}(s)}{Z_{1}(s)+Z_{C}(s)} E_{1}(s)+\frac{2 Z_{C}(s)}{Z_{2}(s)+Z_{C}(s)} E_{2}(s)\right] }
\end{aligned}
$$




$$
\begin{aligned}
& W_{2}(s)=\frac{P(s)}{1-\Gamma_{1}(s) \Gamma_{2}(s) P^{2}(s)} \\
& \cdot\left[\frac{2 Z_{C}(s)}{Z_{1}(s)+Z_{C}(s)} E_{1}(s)+\Gamma_{1}(s) P(s) \frac{2 Z_{C}(s)}{Z_{2}(s)+Z_{C}(s)} E_{2}(s)\right] \\
& P(s)=e^{-s t}
\end{aligned}
$$

$t$ is the travel time of traveling wave in transmission line, $\Gamma_{1}$ (s)and $\Gamma_{2}(\mathrm{~s})$ are the voltage reflection coefficients in 11'and 22':

$$
\begin{gathered}
\Gamma_{1}(s)=\frac{Z_{1}(s)-Z_{C}(s)}{Z_{1}(s)+Z_{C}(s)} \\
\Gamma_{2}(s)=\frac{Z_{2}(s)-Z_{C}(s)}{Z_{2}(s)+Z_{C}(s)}
\end{gathered}
$$

The natural frequency $\omega$ is the roots of the equation:

$$
1-\Gamma_{1}(s) \Gamma_{2}(s) P^{2}(s)=0
$$

Equation (8) has an infinite number of roots, and they are the solution of the equation

$$
\begin{gathered}
1-A_{1} A_{2} e^{i \theta_{1}} e^{i \theta_{2}} e^{-2 \omega t}=0 \\
A_{1} A_{2} e^{i \theta_{1}} e^{i \theta_{2}} e^{2 k \pi i}=e^{2 \omega t} \quad k=0, \pm 1, \pm 2, \ldots,
\end{gathered}
$$

where

$$
\begin{gathered}
A_{1}=\left|\Gamma_{1}\right| \\
A_{2}=\left|\Gamma_{2}\right| \\
\theta_{1}=\operatorname{angle}\left(\Gamma_{1}\right) \\
\theta_{2}=\operatorname{angle}\left(\Gamma_{2}\right)
\end{gathered}
$$

From which we obtain

$$
\omega=\frac{1}{t} \ln \left(\sqrt{A_{1} A_{2}}\right)+i\left(\frac{\theta_{1}+\theta_{2}+2 k \pi}{2 t}\right) k=0, \pm 1, \pm 2, \ldots
$$

$$
f=\frac{\operatorname{imag}(\omega)}{2 \pi}=\frac{\theta_{1}+\theta_{2}+2 k \pi}{2 t \times 2 \pi} \quad k=0, \pm 1, \pm 2, \ldots
$$

These frequencies are called natural frequency of transmission line. If $Z_{1}$ and $Z_{2}$ are zero, natural frequencies satisfy the condition:

$$
\omega=n \omega_{0} \quad n=1,2,3, \ldots
$$

$$
f_{k}=k f_{0} k=1,2,3 \ldots
$$

where

$$
\omega_{0}=\frac{\pi}{t}
$$

Figure II shows the spectrum of natural frequency component after fault. It is clear that natural frequencies are discrete, and they are multiple of main natural frequency.

When the system is in normal condition, there is no natural frequency component, so its spectrum is zero.

\section{B. Spectrum Characteristics of Natural Frequency Component When External Fault}

Figure III shows a four-side-MMC-HVDC system, F1 and F2 is fault points on lines. Relays are installed at two sides of line.

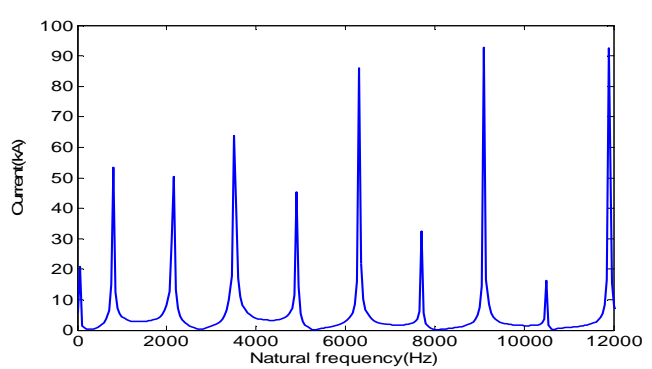

FIGURE II. NATURAL FREQUENCY COMPONENT SPECTRUM

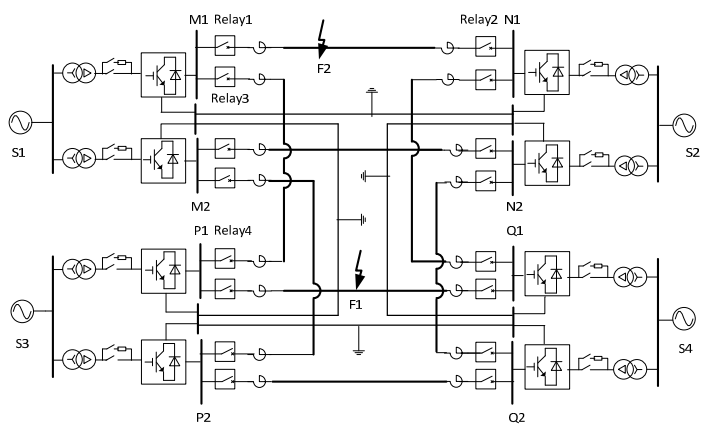

FIGURE III. MMC-HVDC SYSTEM

When a fault occurs at F1, it is an external fault for line MN. The natural frequencies of currents flowing through relay 1 and 2 are the same, so the natural frequency component spectrums of relay 1 and 2 are similar. Fig. IV shows the spectrums of 
natural frequency component in relay 1 and 2 when the fault is in F1. It can be seen from it that the spectrums in two sides of line $\mathrm{MN}$ are similar and the natural frequency component amplitudes of relay 1 are smaller than that of relay 2 , this is the result of natural frequency component decayed.
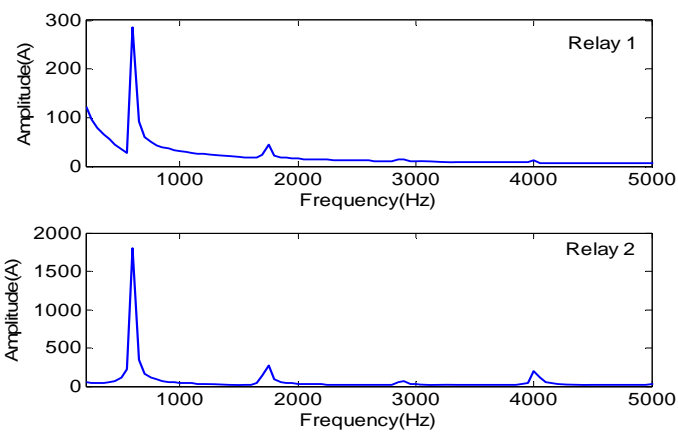

FIGURE IV. CURRENT SPECTRUMS OF NATURAL FREQUENCY COMPONENT DURING EXTERNAL FAULT

\section{Spectrum Characteristics of Natural Frequency Component When Internal Fault}

When a fault occurs at F2, it is an internal fault for line MN, systems in two sides of fault point are different and the natural frequencies of currents flowing through relay 1 and 2 are different. Figure $\mathrm{V}$ shows the natural frequency component spectrums in relay 1 and 2 when the fault is in F2. It can be seen from it that the spectrums in two ends of line $\mathrm{MN}$ are different.
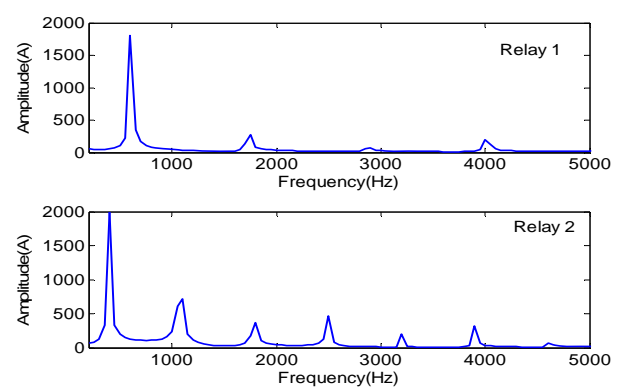

FIGURE V. CURRENT SPECTRUMS OF NATURAL FREQUENCY COMPONENT DURING INTERNAL FAULT

\section{Protection Algorithm BASED ON SPECtRUM CHARACTERISTIC OF NATURAL FREQUENCY COMPONENT}

\section{A. Basic Principle}

According to the analysis above, the spectrum characteristic of natural frequency component can be used to identify internal and external faults. If the spectrums of natural frequency component in two ends of transmission line are similar, the fault is an external fault, and if their difference is great, the fault is an internal fault.

In this paper, the relationship between current spectrums of natural frequency component is expressed by spectrum correlation coefficient and the formula is [11]:

$$
\rho=\frac{\operatorname{cov}(X, Y)}{\sqrt{\sigma^{2}(X) \times \sigma^{2}(Y)}}
$$

and $\operatorname{cov}(X, Y), \sigma^{2}(X)$ and $\sigma^{2}(X)$ are defined by

$$
\begin{gathered}
\operatorname{cov}(X, Y)=\sum_{n=0}^{N} x(n) y(n) \\
\sigma^{2}(X)=\mathrm{E}(X)=\sum_{n=0}^{N} x^{2}(n) \\
\sigma^{2}(Y)=\mathrm{E}(Y)=\sum_{n=0}^{N} y^{2}(n)
\end{gathered}
$$

where $\rho$ is spectrum correlation coefficient of phase $\mathrm{X}$ and phase $\mathrm{Y}, \mathrm{E}(X)$ and $\mathrm{E}(Y)$ are spectrum energy of phase $\mathrm{X}$ and phase Y, $x(n), y(n)$ is sampling value of current spectrum of phase $\mathrm{X}, \mathrm{Y}$ respectively, $N$ is sampling point number in a data window.

If the spectrums of phase $\mathrm{X}$ and phase $\mathrm{Y}$ are similar, $\rho$ is near to 1 , when their spectrums are the same, $\rho$ is equal to 1 . If there is larger difference between spectrums of phase $\mathrm{X}$ and $\mathrm{Y}, \rho$ is near to 0 .

For an external fault, spectrum correlation coefficient of natural frequency component between two ends of line satisfies

$$
\rho>\delta
$$

where $\delta$ is a threshold to identify internal or external fault.

For an internal fault, spectrum correlation coefficient of natural frequency component between fault phases of two ends of fault line satisfies

$$
\rho<\delta
$$

\section{B. Influencing Factor}

\section{1) Fault location}

When the fault point is close to bus $\mathrm{M}$, natural frequency amplitude may be small and its spectrum is nearby 0 , so $\rho$ is near to 0 , the fault can be identified as an internal fault.

When the fault is in the middle of line, spectrums of natural frequency component in two sides of fault point are similar and $\rho$ may be bigger than $\delta$, in this condition, the scheme will fail.

\section{2) Noise}

Compare to natural frequency component, the amplitude of noise is smaller. It can be filtered through setting a threshold. 


\section{3) Fault resistance}

The scheme can identify fault phase in high resistance condition. But if the fault resistance is too high, the sensitivity of scheme will reduce.

4) Window length, the sampling time and the maximum frequency of the spectrum

For spectrum by FFT, the maximum frequency of the spectrum is relation to the window length and the sampling time (sampling frequency). The maximum frequency of the spectrum is half of sampling frequency. The window length is bigger than $1 / f_{\max }$ and $f_{\max }$ is the maximum frequency of the spectrum.

\section{5) Switching of circuit breakers}

For line charging power, the current in one side of line is capacitance current and the current in other side is zero. In this case, $\rho$ is near to 0 . Adding criterion $I>I_{\text {set, }}$ if $I<I_{\text {set, }}$ the protection scheme is locked.

\section{Protection Scheme}

The flowchart of protection scheme base on spectrum characteristic of natural frequency component can be described as following:

1) Sample currents of two ends of line, then compute the spectrum respectively.

2) Set a threshold value $\delta_{1}$ which is used to eliminate noise, if the amplitude of frequency in spectrum is smaller than $\delta_{1}$, remove it from spectrum. If all frequency amplitudes in one end of line are smaller than $\delta_{1}$, let the spectrum be zero. If all amplitudes of frequency in two ends of line are smaller than $\delta_{1}$, turn step 4).

3) Compute the spectrum correlation coefficient $\rho$ ( $\rho$ is between 0 and 1), then set $\delta_{2}$ which is used to identify internal fault and other conditions, compare $\rho$ and $\delta_{2}$, if $\rho<\delta_{2}$, the fault is an internal fault. If $\rho>\delta_{2}$, turn step 4).

4) Other methods are used to identify internal and external faults.

\section{Simulation And Scheme Response Evaluation}

The structure of simulation system is shown in Fig. 3. The parameters of source and transmission line are given in Appendix. Relays are located at two sides of lines. The sampling rate is $50 \mathrm{k} \mathrm{Hz}$. FFT is used to compute current spectrums. The values of $\delta_{1}, \delta_{2}$ are $100 \mathrm{~A}, 0.7$ respectively. The current signals from each ends of transmission lines are determined using PSCAD/EMTDC simulation environment.

$\mathrm{MN} 500 \mathrm{kV}$ line ground fault in three typical fault positions (outlet, middle and end of line $\mathrm{MN}$ ) were considered respectively to evaluate the influence of fault position for protection scheme. Table I shows $\rho$ of line MP and PN when A-B-short occurs in different points.
Figure VI shows the spectrum of relay 1 4 when fault is at outlet of bus M. It can be seen that the spectrum of relay land 2 are different, the spectrum of relay 1 and 2 is the same.

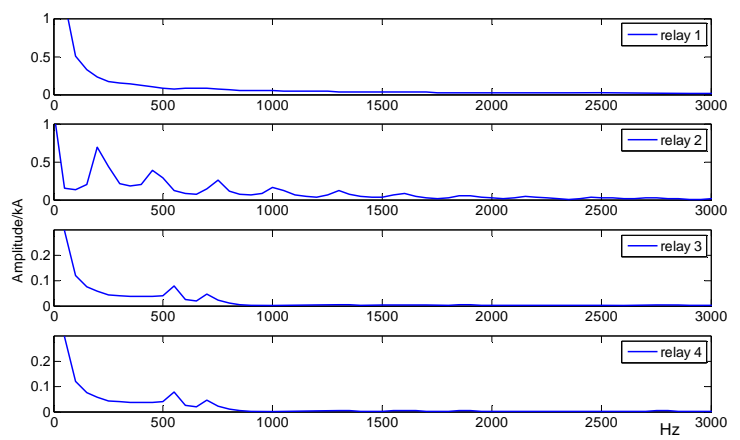

FIGURE VI. CURRENT SPECTRUMS OF NATURAL FREQUENCY COMPONENT OF RELAY 1 4

Table I shows the spectrum correlation coefficients according (20), it can be seen that it is near to 1 for external faults. When faults are near two sides of line $\mathrm{MN}$, spectrum correlation coefficients are much smaller than 1. Obviously the scheme can identify internal fault nearby bus.

TABLE I. SPECTRUM CORRELATION COEFFICIENTS OF FAULT PHASE WHEN GROUND FAULTS IN DIFFERENT FAULT LOCATIONS

\begin{tabular}{c|c|c}
\hline Fault Location & $\rho(\mathrm{MN})$ & $\rho(\mathrm{MP})$ \\
\hline Outlet of MN & 0.08067 & 0.998 \\
\hline Middle of MN & 0.98 & 1 \\
\hline End of MN & 0.06776 & 0.998 \\
\hline
\end{tabular}

When a fault is in the middle point of transmission line $\mathrm{MN}$, natural frequencies on two sides of fault point are similar, $\rho$ is near to 1 . But the current polarities of relay 1 and 2 are the same and the current polarities of relay 3 and 4 are different (shown in Figure VII). So the scheme can identify faults of MMC-HVDC line.

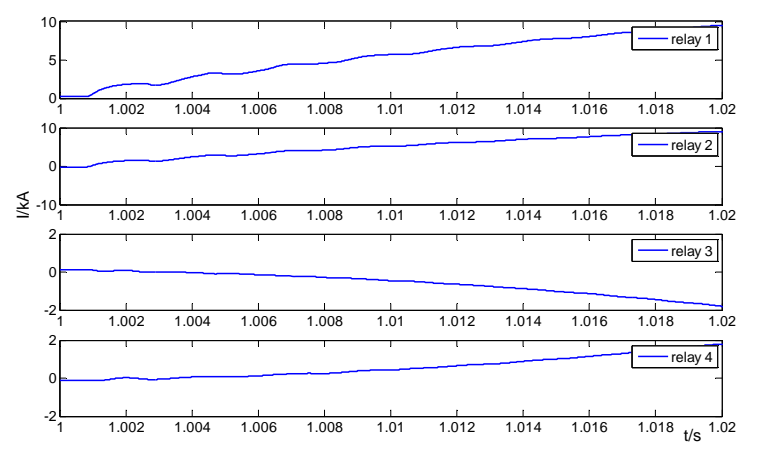

FIGURE VII. CURRENT OF RELAY 1 4 WHEN THE FAULT IS AT MIDDLE OF LINE MN

\section{APPENDIX}

The system is a $\pm 500 \mathrm{kV}$ transmission lines.

\begin{tabular}{|l|l|l|l|l|}
\hline & S4 & S3 & S1 & S2 \\
\hline Max Power DC/MW & 3000 & 3000 & 1500 & 1500 \\
\hline Module capacitance $/ \mathrm{mF}$ & 15 & 15 & 10 & 10 \\
\hline Module Number & 240 & 240 & 240 & 240 \\
\hline
\end{tabular}


Transmission line parameters (Bergeron Model):

$\mathrm{MN}=500 \mathrm{~km}, \quad \mathrm{NQ}=500 \mathrm{~km}, \quad \mathrm{MP}=180 \mathrm{~km}, \quad \mathrm{PQ}=500 \mathrm{~km}$, $R_{1}=0.00758 \Omega / \mathrm{km}, \quad X_{1}=0.26365 \Omega / \mathrm{km}, \quad C_{1}=0.01397 \mu \mathrm{F} / \mathrm{km}$, $\mathrm{R}_{0}=0.15421 \Omega / \mathrm{km}, X_{0}=0.8306 \Omega / \mathrm{km}, C_{0}=0.009296 \mu \mathrm{F} / \mathrm{km}$.

\section{REFERENCES}

[1] A. G. Phadke and J. S. Trorp, Computer Relaying for Power Systems. Philadelphia, PA: Research Studies Press, 1988, pp. 112-164.

[2] T. Takagi, J. Baba, K. Uemura and T. Sakagushi, "Fault Protection Based on Traveling Wave Theory, Part I: Theory", IEEE PES Summer Meeting, July 1977, pp.750-753.

[3] H. W. Dommel, J. M. Michels, "High Speed Relaying Using Traveling Wave Transient Analysis", 1978 PES Winter Meeting, New York, Jan. 1978, No.A78, pp.1-7.

[4] M. Chamia, S. Liberman, "Ultra High Speed Relay for EHV/UHV Transmission lines: Development, Design and Application”, IEEE Trans. $P A S-97$, No.5, May 1978, pp.2104-2112.

[5] K. S. Prakash, "Amplitude Comparator Based Algorithm for Directional Comparison Protection of Transmission Lines", IEEE PES Winter Meeting, New York, 1989, pp525-677.

[6] P. A. Crossley, P. G. Mclaren, "Distance protection based on traveling wave", IEEE Trans.on PAS-102, N0.9, Sep.1983, pp.2971-2983.

[7] C. Christo, D. W. P. Thomas, W. Arther, "Scheme, Based on Traveling Wave, for the Protection of Major Transmission Lines", IEE Proceeding, Vol.135,No.1, Jan.1988, pp.63-73.

[8] X. Z. Dong, Y. Z. Ge, B. Y. Xu, "A New Device for Fault Location of Transmission Lines", Power System Technology, Vol.22,No.1, Jan.1998, pp. $17-21$

[9] E. H. Shehab-Eldin and P. G. Mclaren, "Traveling wave distance protection-problem areas and solutions," IEEE Trans. Power Del., vol.3, no. 3, pp. 894-902, Jul. 1988.

[10] M. Giovanni, Transmission lines and lumped circuits [M]. Academic Press, San Diego, USA, 2001, pp.72-79.

[11] J. C. Cheng, J. Zhang, Z. P. Wang, "Identification of the transient faults occurring on the extra high voltage transmission lines based on correlation analysis and its improved algorithm." Automation of Electric Power System, vol. 29, no.3, Feb. 2005, pp.26-30 